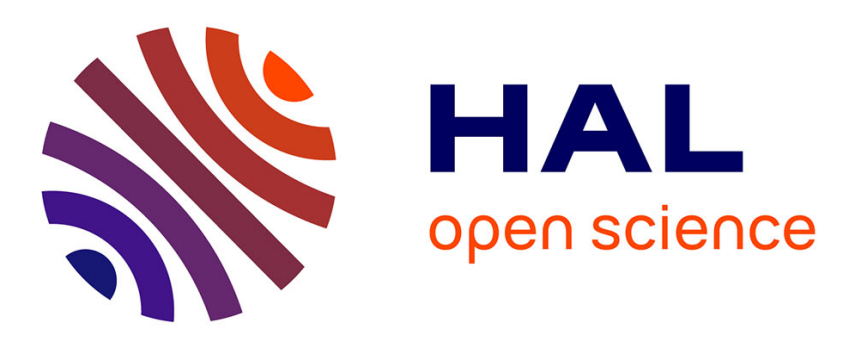

\title{
Multi-dimensional harmonic balance applied to rotor dynamics
}

Mikhail Guskov, Jean-Jacques Sinou, Fabrice Thouverez

\section{To cite this version:}

Mikhail Guskov, Jean-Jacques Sinou, Fabrice Thouverez. Multi-dimensional harmonic balance applied to rotor dynamics. Mechanics Research Communications, 2008, 35, pp.537-545. 10.1016/j.mechrescom.2008.05.002 . hal-00322891

\section{HAL Id: hal-00322891 https://hal.science/hal-00322891}

Submitted on 26 Sep 2012

HAL is a multi-disciplinary open access archive for the deposit and dissemination of scientific research documents, whether they are published or not. The documents may come from teaching and research institutions in France or abroad, or from public or private research centers.
L'archive ouverte pluridisciplinaire HAL, est destinée au dépôt et à la diffusion de documents scientifiques de niveau recherche, publiés ou non, émanant des établissements d'enseignement et de recherche français ou étrangers, des laboratoires publics ou privés. 


\title{
Multi-dimensional harmonic balance applied to rotor dynamics
}

\author{
Mikhail Guskov, Jean-Jacques Sinou and Fabrice Thouverez \\ Laboratoire de Tribologie et Dynamique des Systèmes UMR-CNRS 5513 \\ Ecole Centrale de Lyon, 36 avenue Guy de Collongue \\ 69134 Ecully Cedex, France
}

\begin{abstract}
The multi-frequency dynamical behavior is a feature of many technical systems. Multiple shaft rotating machinery, subject to simultaneous unbalances spinning at different speeds is a particular case of such systems. Common methods of steady state solution are not valid when the addressed systems have non-linear properties. This study presents a generalized version of harmonic balance coupled with arc-length continuation, developed in order to study non-linear responses of modified Jeffcott rotor system subject to multiple unbalances. Numerically, the non-linear terms are taken into account via an AFT (alternating frequency-time domain) procedure, involving multi-dimensional FFT. An improvement to the AFT approach is proposed. The example addressed in this paper is a modified Jeffcott rotor with piece-wise radial stiffness.
\end{abstract}

\section{INTRODUCTION}

Numerous technical systems are subject to multi-frequency excitation: gears, multi-spool aircraft engines, electrical circuits. The excitation in these systems is composed of several harmonic terms with frequencies which have not necessarily integer or even rational ratio between them. In case of presence of non-linearities, conventional frequency domain methods are unable to provide the dynamical response for such cases. One of the approaches to treat multi-frequency systems is a generalization of the harmonic balance in rotor systems [SL07, PM97] for multi-dimensional time and frequency domains.

Chua and Ushida [CU81] have presented in 1981 a generalization of the harmonic balance method in application to multi-frequency electrical circuits. Non-linear terms have been processed by trigonometrical collocation-type procedure. In 1983 Lau et Cheung [LC83] have adjusted his method to incremental harmonic balance formulation. In 1996 and 1997, this method was further developed by Kim and Noah through coupling it with an AFT (alternative time-frequency domain) approach and using FFT [KN96, KC97]. Pusenjak and Oblak [PO04] have presented in 2004 a multi-dimensional harmonic balance method with arc-length continuation.

Multiple-rotor systems with intershaft bearings, such as aircraft engines, derived equipment or test rigs, are subject to multi-frequency excitation due to several unbalances present simultaneously [Hib75, LF97, Ehr98, GSTN06]. One of sources of non-linearities in such systems is the utilization of rolling bearings that implies radial clearance and non-linear contact stiffness [Har66, VST08, GSTN07].

In this study we will address the non-linear rotordynamics of a modified Jeffcott rotor model under quasi-periodic excitation symbolizing the presence of a dual unbalance. The dynamic response of this 
system is computed by the numerical algorithm based on a multi-dimensional generalization of harmonic balance and continuation. A conventional harmonic balance method is first presented in view of introducing the framework, providing a basis for further generalization and proposing an improvement to the AFT technique. After the presentation of the numerical method, we consider the influence of the presence of two components of the excitation on the unbalance response of the studied system.

\section{NUMERICAL METHOD: GENERALIZED HARMONIC BALANCE}

Equation of motion. The method developed in this study addresses structural dynamics systems, described by the following matricial equation of size $n$ :

$$
\mathbf{M} \ddot{\mathbf{x}}+\mathbf{D} \dot{\mathbf{x}}+\mathbf{K} \mathbf{x}+\mathbf{f}_{\mathrm{NL}}(\mathbf{x})=\mathbf{g}(t)
$$

where $\mathbf{M}, \mathbf{D}$, and $\mathbf{K}$ stand for mass, generalized damping and stiffness matrices respectively, $\mathbf{x}, \mathbf{f}_{\mathrm{NL}}, \mathbf{g}$, mean generalized displacements, non-linear terms and excitation vectors respectively, $n$ is the number of degrees of freedom.

Conventional harmonic balance. A great deal of technical systems feature periodic oscillatory behavior that can be expressed by simple Fourier series:

$$
\mathbf{x}(t)=\sum_{k=0}^{\infty} \tilde{\mathbf{a}}_{k} \cos k \omega t+\tilde{\mathbf{b}}_{k} \sin k \omega t
$$

Here the tilde sign ( $)$ is used to denote the frequency domain variables. The presence of a particular frequency makes it interesting to introduce a normalized time variable

$$
\tau=\omega t
$$

A number of applied methods exists for extracting motions of the form (2), the harmonic balance is one of the most spread.

By the following, the inverse discrete Fourier transform (2) will be denoted by means of the matrix $\mathbf{T}(\tau)$ composed of sines and cosines up to order $N$, which produces $N_{H}=2 N+1$ harmonic components:

$$
\mathbf{x}(\tau)=\mathbf{T}(\tau) \tilde{\mathbf{x}},
$$

where the vector $\tilde{\mathbf{x}}$ (size $N_{H} n$ ) is composed of the components of $\tilde{\mathbf{a}}$ and $\tilde{\mathbf{b}}$ in accordance with $\mathbf{T}$

The substitution of the approximation (4) in the equation of motion (1), followed by a Galerkin projection,

$$
\int_{0}^{2 \pi} \mathbf{T}^{\mathrm{T}}\left(\omega^{2} \mathbf{M} \frac{\partial^{2} \mathbf{T}}{\partial \tau^{2}} \tilde{\mathbf{x}}+\omega \mathbf{D} \frac{\partial \mathbf{T}}{\partial \tau} \tilde{\mathbf{x}}+\mathbf{K} \mathbf{T} \tilde{\mathbf{x}}+\tilde{\mathbf{f}}_{\mathrm{NL}}(\mathbf{T} \tilde{\mathbf{x}})-\mathbf{T} \tilde{\mathbf{g}}\right) d \tau=0
$$

results in a system of non-linear algebraic equations with $\tilde{\mathbf{x}}$ as unknowns:

$$
\mathbf{L} \tilde{\mathbf{x}}+\tilde{\mathbf{f}}_{\mathrm{NL}}(\tilde{\mathbf{x}})-\tilde{\mathbf{g}}=\tilde{\mathbf{r}}(=0) .
$$


Here $\mathbf{L}$ stands for the linear part of the equation (dynamic stiffness corresponding to each harmonic component):

$$
\begin{gathered}
\mathbf{L}=\int_{0}^{2 \pi} \mathbf{T}^{\mathrm{T}}\left(\omega^{2} \mathbf{M} \frac{\partial^{2} \mathbf{T}}{\partial \tau^{2}}+\omega \mathbf{D} \frac{\partial \mathbf{T}}{\partial \tau}+\mathbf{K} \mathbf{T}\right) d \tau=\left[\begin{array}{cccc}
\mathbf{L}^{\{0\}} & 0 & \ldots & 0 \\
0 & \mathbf{L}^{\{1\}} & \ldots & 0 \\
\vdots & \vdots & \ddots & \vdots \\
0 & 0 & \ldots & \mathbf{L}^{\{N\}}
\end{array}\right], \\
\mathbf{L}^{\{0\}}=\mathbf{K} ; \quad \mathbf{L}^{\{j\}}=\left[\begin{array}{cc}
\mathbf{K}-(j \omega)^{2} \mathbf{M} & j \omega \mathbf{D} \\
-j \omega \mathbf{D} & \mathbf{K}-(j \omega)^{2} \mathbf{M}
\end{array}\right], \quad j=1, \ldots, N,
\end{gathered}
$$

$\tilde{\mathrm{g}}$ and $\tilde{\mathbf{f}}$ correspond to excitation end non-linear terms:

$$
\tilde{\mathbf{g}}=\int_{0}^{2 \pi} \mathbf{T}^{\mathrm{T}} \mathbf{g} d \tau, \quad \tilde{\mathbf{f}}_{\mathrm{NL}}(\tilde{\mathbf{x}})=\int_{0}^{2 \pi} \mathbf{T}^{\mathrm{T}} \mathbf{f}_{\mathrm{NL}}(\mathbf{T} \tilde{\mathbf{x}}) d \tau .
$$

The non-linear algebraic equation (6) can be solved by a Newton-like method. The non-linear terms $\tilde{\mathbf{f}}_{\mathrm{NL}}$ as well as their derivatives $\frac{\partial \tilde{\mathrm{f}}_{\mathrm{N} L}}{\partial \tilde{\mathbf{x}}_{\mathrm{x}}}$ can be computed in the time domain by an AFT procedure. FFT may be used for the passage from the time domain to the frequency domain:

$$
\tilde{\mathbf{x}} \stackrel{\mathbf{T} \tilde{\mathbf{x}}}{\longrightarrow} \mathbf{x}(t) \rightarrow \mathbf{f}_{\mathrm{NL}}(\mathbf{x}) \stackrel{\text { FFT }}{\longrightarrow} \tilde{\mathbf{f}}_{\mathrm{NL}}(\tilde{\mathbf{x}}) .
$$

As for the derivatives matrix, it can be constructed column by column:

$$
\left[\frac{\partial \tilde{\mathbf{f}}_{\mathrm{NL}}}{\partial \tilde{\mathbf{x}}}\right]_{i j}=\frac{\left[\tilde{\mathbf{f}}_{\mathrm{NL}}\left(\tilde{\mathbf{x}}+\mathbf{e}_{j} \Delta x\right)-\tilde{\mathbf{f}}_{\mathrm{NL}}(\tilde{\mathbf{x}})\right]_{i}}{\Delta x}, \quad i, j=1, \ldots, N_{H} n .
$$

with $i, j$ indicating row and column in the matrix, $\mathbf{e}_{i}$ unit vector in $i$ th direction and $\Delta x$ small increment. When the number of non-linear DOFs $n_{\mathrm{NL}}$ is smaller than $n$, the computation of (11) may be restricted to the respective columns and lines. The number of FFT calculations implied in such a procedure is proportional to $\left(N_{H} n_{\mathrm{NL}}\right)^{2}$.

This approach, limited to the periodic motions, can be extended to a broader class of problems.

Multi-dimensional time and frequency domain. Many types of technical systems are known to have such dynamical behavior that can be represented by multiple Fourier series with $M>1$ basic frequencies:

$$
\mathbf{x}(t)=\sum_{\mathbf{k} \in \mathbb{Z}^{M}}^{\infty} \tilde{\mathbf{a}}_{\mathbf{k}} \cos (\mathbf{k}, \boldsymbol{\omega}) t+\tilde{\mathbf{b}}_{\mathbf{k}} \sin (\mathbf{k}, \boldsymbol{\omega}) t, \quad \boldsymbol{\omega}=\left[\omega_{1}, \ldots, \omega_{M}\right]^{T} \in \mathbb{R}^{M}
$$

Here the vector $\boldsymbol{\omega}$ is the frequency basis, $\mathbf{k}$ stands for the vectorial harmonic indexes and $($,$) denotes$ the scalar product. One of the approaches to such functions is to consider $\mathbf{x}(t)$ as an equivalent function $\overline{\mathbf{x}}(\tau)$ of $M$ time variables in a $M$-dimensional time domain $\tau=\left[\tau_{1}, \ldots \tau_{M}\right]=\boldsymbol{\omega} t, 2 \pi$-periodic in each component of the hyper-time $\tau$ :

$$
\mathbf{x}(t)=\overline{\mathbf{x}}(\tau)=\overline{\mathbf{x}}(\boldsymbol{\omega} t)
$$




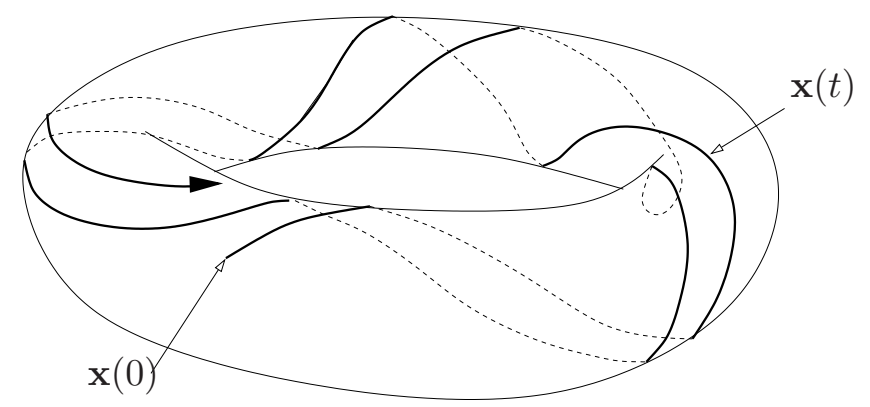

Figure 1: HYPERTIME CONCEPTS RELATED TO A BI-PERIODIC MOTION

The time derivatives involved by such hyper-time variables are reformulated as follows:

$$
\dot{\mathbf{f}}=\frac{\partial \mathbf{f}}{\partial t}=\frac{\partial \overline{\mathbf{f}}}{\partial \boldsymbol{\tau}} \frac{\partial \boldsymbol{\tau}}{\partial t}=\frac{\partial \overline{\mathbf{f}}}{\partial \boldsymbol{\tau}} \boldsymbol{\omega}
$$

For $M=2$, the bi-periodic motion (12) covers the invariant torus in the phase space (Fig. 1). For the sake of clarity of the presentation, the bar sign referring to the multi-dimensional time domain will be omitted by the following. This generalization may as well be performed with the series representation (12). The truncation to the harmonic $N$ implies the presence of $N_{H}$ harmonics:

$$
N_{H}=\frac{(2 N+1)^{M}+1}{2}
$$

Generalized harmonic balance. By applying the harmonic representation (12) to the equation of motion (1) and by projecting it on the same harmonic functions by a Galerkin procedure analogous to (5), we obtain the harmonic balance equation similar to (6):

$$
\mathbf{L} \tilde{\mathbf{x}}+\tilde{\mathbf{f}}_{\mathrm{NL}}(\tilde{\mathbf{x}})-\tilde{\mathbf{g}}=\tilde{\mathbf{r}}(=0) ;
$$

where $\mathbf{L}$ is modified for the new harmonic contents considered:

$$
\begin{gathered}
\mathbf{L}=\left[\begin{array}{cccc}
\mathbf{L}^{\{0\}} & 0 & \ldots & 0 \\
0 & \mathbf{L}^{\left\{\mathbf{k}_{1}\right\}} & \ldots & 0 \\
\vdots & \vdots & \ddots & \vdots \\
0 & 0 & \ldots & \mathbf{L}^{\left\{\mathbf{k}_{N_{H}-1}\right\}}
\end{array}\right], \\
\mathbf{L}^{\{0\}}=\mathbf{K}, \quad \mathbf{L}^{\left\{\mathbf{k}_{j}\right\}}=\left[\begin{array}{cc}
\mathbf{K}-\left(\mathbf{k}_{j}, \boldsymbol{\omega}\right)^{2} \mathbf{M} & \left(\mathbf{k}_{j}, \boldsymbol{\omega}\right) \mathbf{D} \\
-\left(\mathbf{k}_{j}, \boldsymbol{\omega}\right) \mathbf{D} & \mathbf{K}-\left(\mathbf{k}_{j}, \boldsymbol{\omega}\right)^{2} \mathbf{M}
\end{array}\right], \quad j=1, \ldots, N_{H}-1 .
\end{gathered}
$$

The AFT approach can be generalized by the use of $M$-dimensional FFT so as to apply to the $M$ dimensional hyper-time period.

As compared to the approach, described by (11) a way to accelerate the computation of the derivatives matrix can be developed by taking advantage of the correlated nature of the coefficients of Fourier series for $\mathbf{f}_{\mathrm{NL}}$ and $\tilde{\mathbf{x}}$. To do so in terms of FFT, consider the following complex representation:

$$
\overline{\mathbf{x}}_{(j)}=\frac{1}{p} \sum_{l=0}^{p-1} e^{\frac{2 \pi i l j}{p}}\left(\tilde{\mathbf{a}}^{\{l\}}-i \tilde{\mathbf{b}}^{\{l\}}\right),
$$




$$
\overline{\mathbf{f}}_{(j)}=\frac{1}{p} \sum_{l=0}^{p-1} e^{\frac{2 \pi i l j}{p}}\left(\tilde{\mathbf{c}}^{\{l\}}-i \tilde{\mathbf{d}}^{\{l\}}\right) .
$$

The derivatives of the terms of $k$ th harmonic term of $\tilde{\mathbf{f}}_{\mathrm{NL}}$ with respect to the and $l$ th harmonic term of $\tilde{\mathbf{x}}$ read as follows

$$
\frac{\partial \tilde{\mathbf{f}}_{\mathrm{NL}}^{\{k\}}}{\partial \tilde{\mathbf{x}}^{\{l\}}}=\sum_{j=0}^{p-1} e^{-\frac{2 \pi i k j}{p}}\left[\frac{\partial \mathbf{f}_{\mathrm{NL}}}{\partial \mathbf{x}}\right]_{\mathbf{x}=\overline{\mathbf{x}}_{(j)}} \frac{\partial \overline{\mathbf{x}}_{(j)}}{\partial \tilde{\mathbf{x}}^{\{l\}}},
$$

or, in a more detailed way, consider $i$ th DOF of $\tilde{\mathbf{f}}_{\mathrm{NL}}$ and $j$ th DOF of $\tilde{\mathbf{x}}_{\mathrm{NL}}$ :

$$
\begin{aligned}
& \frac{\partial \tilde{\mathbf{c}}_{[i]}^{\{k\}}}{\partial \tilde{\mathbf{a}}_{[j]}^{\{l\}}}=\frac{1}{p} \sum_{q=0}^{p-1} \cos \left(\frac{2 \pi k q}{p}\right)\left[\frac{\partial \mathbf{f}_{\mathrm{NL}[i]}}{\partial \mathbf{x}_{[j]}}\right]_{\mathbf{x}=\overline{\mathbf{x}}_{(q)}} \cos \left(\frac{2 \pi q l}{p}\right) ; \\
& \frac{\partial \tilde{\mathbf{c}}_{[i]}^{\{k\}}}{\partial \tilde{\mathbf{b}}_{[j]}^{\{l\}}}=\frac{1}{p} \sum_{q=0}^{p-1} \cos \left(\frac{2 \pi k q}{p}\right)\left[\frac{\partial \mathbf{f}_{\mathrm{NL}[i]}}{\partial \mathbf{x}_{[j]}}\right]_{\mathbf{x}=\overline{\mathbf{x}}_{(q)}} \sin \left(-\frac{2 \pi q l}{p}\right) ; \\
& \frac{\partial \tilde{\mathbf{d}}_{[i]}^{\{k\}}}{\partial \tilde{\mathbf{a}}_{[j]}^{\{l\}}}=\frac{1}{p} \sum_{q=0}^{p-1} \sin \left(\frac{2 \pi k q}{p}\right)\left[\frac{\partial \mathbf{f}_{\mathrm{NL}[i]}}{\partial \mathbf{x}_{[j]}}\right]_{\mathbf{x}=\overline{\mathbf{x}}_{(q)}} \cos \left(\frac{2 \pi q l}{p}\right) ; \\
& \frac{\partial \tilde{\mathbf{d}}_{[i]}^{\{k\}}}{\partial \tilde{\mathbf{b}}_{[j]}^{\{l\}}}=\frac{1}{p} \sum_{q=0}^{p-1} \sin \left(\frac{2 \pi k q}{p}\right)\left[\frac{\partial \mathbf{f}_{\mathrm{NL}[i]}}{\partial \mathbf{x}_{[j]}}\right]_{\mathbf{x}=\overline{\mathbf{x}}_{(q)}} \sin \left(-\frac{2 \pi q l}{p}\right)
\end{aligned}
$$

with $i, j=1, \ldots, n$ and $k, l=0, \ldots, N$. By introducing the notation

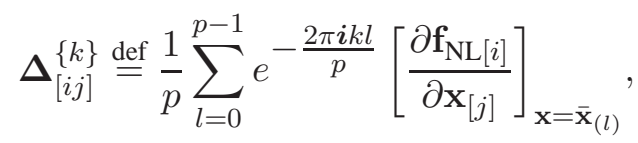

after trigonometric transformations, the relations (22)-(25) can be rewritten as follows

$$
\begin{aligned}
& \frac{\partial \tilde{\mathbf{c}}_{[i]}^{\{k\}}}{\partial \tilde{\mathbf{a}}_{[j]}^{\{l\}}}=\operatorname{Re} \boldsymbol{\Delta}_{[i j]}^{\{k+l\}}+\operatorname{Re} \boldsymbol{\Delta}_{[i j]}^{\{k-l\}} ; \\
& \frac{\partial \tilde{\mathbf{c}}_{[i]}^{\{k\}}}{\partial \tilde{\mathbf{b}}_{[j]}^{\{l\}}}=\operatorname{Im} \boldsymbol{\Delta}_{[i j]}^{\{k+l\}}-\operatorname{Im} \boldsymbol{\Delta}_{[i j]}^{\{k-l\}} ; \\
& \frac{\partial \tilde{\mathbf{d}}_{[i]}^{\{k\}}}{\partial \tilde{\mathbf{a}}_{[j]}^{\{l\}}}=-\operatorname{Im} \boldsymbol{\Delta}_{[i j]}^{\{k+l\}}-\operatorname{Im} \boldsymbol{\Delta}_{[i j]}^{\{k-l\}} ; \\
& \frac{\partial \tilde{\mathbf{d}}_{[i]}^{\{k\}}}{\partial \tilde{\mathbf{b}}_{[j]}^{\{l\}}}=\operatorname{Re} \boldsymbol{\Delta}_{[i j]}^{\{k+l\}}-\operatorname{Re} \boldsymbol{\Delta}_{[i j]}^{\{k-l\}},
\end{aligned}
$$

Thus, the number of FFT computations can be reduced thanks to reutilization of the same transform (26). This technique is particularly interesting when tangent stiffness in time domain is known analytically. 


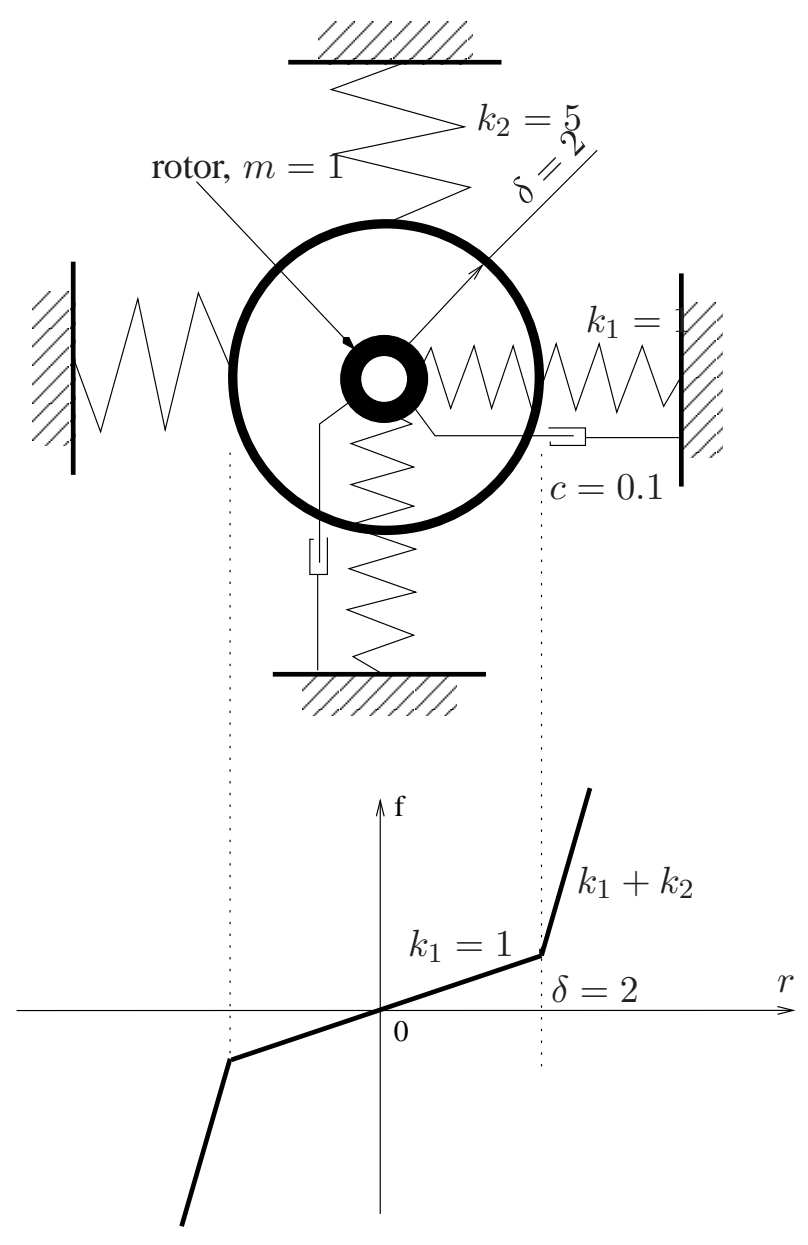

Figure 2: MODIFIED JEFFCOTT ROTOR MODEL

In order to ensure the proper path following on the response curve, arc-length continuation is used. It consists in controlling the length of curvilinear step $\Delta s$ along the response curve $\tilde{\mathbf{x}}(\nu)$. To this end, the solution of the non-linear equation (16) is coupled with an arc-length continuation condition:

$$
\left\|\left[\begin{array}{c}
\tilde{\mathbf{x}} \\
\nu
\end{array}\right]^{j+1}-\left[\begin{array}{c}
\tilde{\mathbf{x}} \\
\nu
\end{array}\right]^{j}\right\|^{2}-\Delta s^{2}=0 .
$$

Here $\nu$ is the control parameter and the superscripts $j, j+1$ denote the numbers of considered consecutive points on the response curve. The step size $\Delta s$ is adjusted according to the convergence of previous steps.

The expression (31) augments the system (16) so one can determine the value of $\nu$. In our study, the control parameter is equal to the spin speed of one of two rotors, the ratio between the two spin speeds is constant. 


\section{NUMERICAL EXAMPLE}

Consider the following elementary 2 DOF example consisted of a modified Jeffcott rotor, mounted in a piecewise-linear pedestal and subject to two unitary unbalances (Fig. 2), rotating at speeds $\omega_{1}, \omega_{2}$ with

$$
\frac{\omega_{1}}{\omega_{2}}=\sqrt{2}
$$

The equation of motion (1) takes the following form for this system:

$$
m\left[\begin{array}{ll}
1 & 0 \\
0 & 1
\end{array}\right]\left[\begin{array}{l}
\ddot{x} \\
\ddot{y}
\end{array}\right]+c\left[\begin{array}{ll}
1 & 0 \\
0 & 1
\end{array}\right]\left[\begin{array}{l}
\dot{x} \\
\dot{y}
\end{array}\right]+k_{1}\left[\begin{array}{ll}
1 & 0 \\
0 & 1
\end{array}\right]\left[\begin{array}{l}
x \\
y
\end{array}\right]+\mathbf{f}_{\mathrm{NL}}(\mathbf{x})=\mathbf{g}(t) .
$$

with the radial piecewise linear stiffness

$$
\mathbf{f}_{\mathrm{NL}}(\mathbf{x})=\left[\begin{array}{c}
h(r-\delta) k_{2}\left(1-\frac{\delta}{r}\right) x \\
h(r-\delta) k_{2}\left(1-\frac{\delta}{r}\right) y
\end{array}\right]
$$

and the external excitation vector composed by two unbalance forces

$$
\mathbf{g}(t)=\omega_{1}^{2}\left[\begin{array}{c}
\cos \omega_{1} t \\
\sin \omega_{1} t
\end{array}\right]+\omega_{2}^{2}\left[\begin{array}{c}
\cos \omega_{2} t \\
\sin \omega_{2} t
\end{array}\right]
$$

$r=\sqrt{x^{2}+y^{2}} ; h($.$) Heaviside's step function; m=1, k_{1}=1, k_{2}=5, c=0.1, \delta=2$ scalar mass, linear and non-linear stiffness, damping and radial clearance parameters.

The system (33) has been solved by multidimensional harmonic balance up to $N=7$ in order to obtain a solution converged with $N$. The comparison between the speed of computations with different AFT procedures ((11) vs. (26-30)) in terms of MATLAB CPU time yields a reduction factor 1.5 to 2 thanks to the proposed approach.

Fig. 2 shows the response curve of this system in terms of the euclidian norm of the vector of harmonic amplitudes $\tilde{\mathbf{x}}$ (DOF 1) versus the excitation frequency $\omega_{1}$ or $s$ (curvilinear co-ordinate, see (31), this presentation "unfolds" the response curve). The key points of the response are denoted by letters A, B, C, D, E in order to associate them between two charts. One can see on the chart two principal peaks (B and D) corresponding to the harmonic components $\{1,0\}$ and $\{0,1\}$, as well as two additional peaks (A and E) due to internal resonances: the components $\{2,-1\}$ and $\{-2,1\}$ are the most excited on these peaks.

A numerical integration has been undertaken in the vicinity of points $A, B, C$ and $D$ in order to test the observability of the obtained solutions (see Fig. 4, HBM: solution by harmonic balance, TM: solution by time marching). As one may remark on all the plots of Fig. 4, the response is not periodic as in case of a conventional harmonic balance solution. The simulations in points $\mathrm{A}$ and $\mathrm{C}$ exhibit significant mismatching between HBM and TM. The point C corresponds to an HBM solution that is "classically" unstable so the TM tends to a stable solution that has another amplitude. The computations for the points B and D (near the top of the peaks, $\|\tilde{\mathbf{x}}\|>25$ ) show a very good resemblance between the HBM and TM results.

Finally, Fig. 5-7 show the coefficients of the harmonic terms of the response. Fig. 5 and 6 show the fundamental terms with respect to each excitation component, i.e. $\tilde{\mathbf{a}}$ and $\tilde{\mathbf{b}}$, in following cases:

- quasi-periodic excitation according to (35),

- periodic excitation corresponding to the first term of (35) only, 


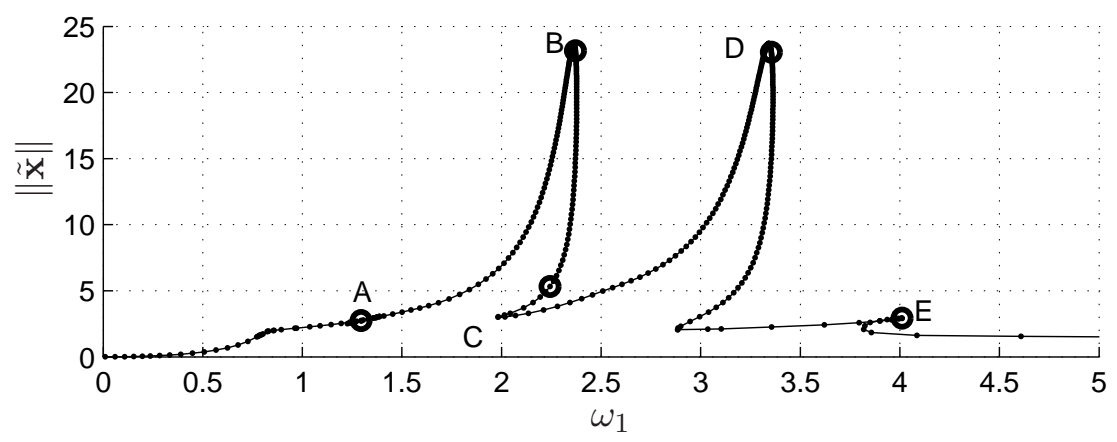

(a) $\|\tilde{\mathbf{x}}\|$ versus $\omega_{1}$

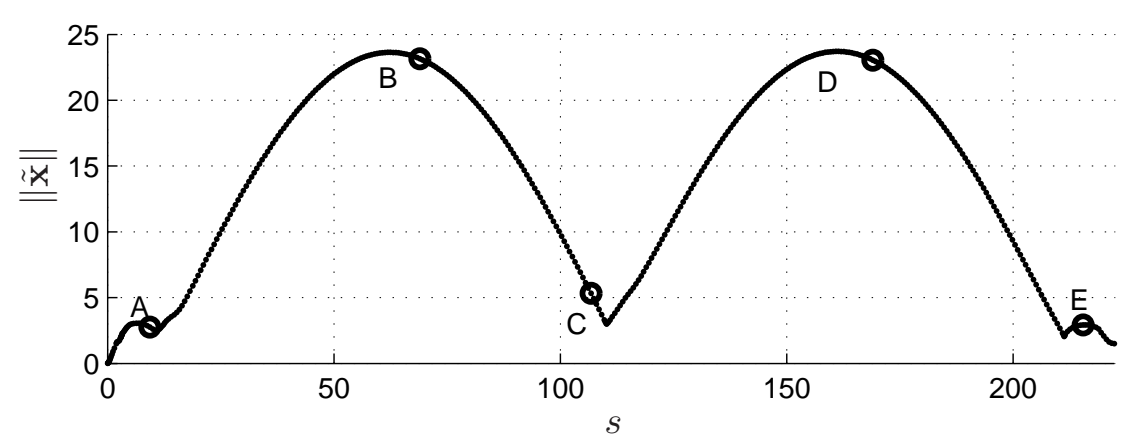

(b) $\|\tilde{\mathbf{x}}\|$ versus $s$

Figure 3: RESPONSE CURVES
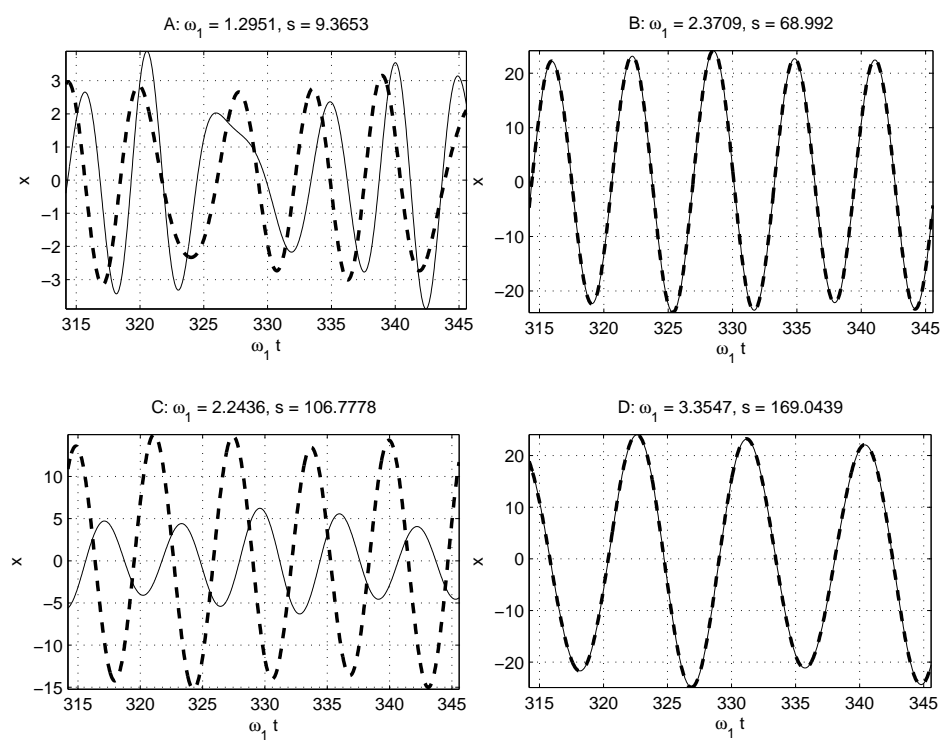

Figure 4: TIME HISTORY DETAIL (- HBM, - - TM) 

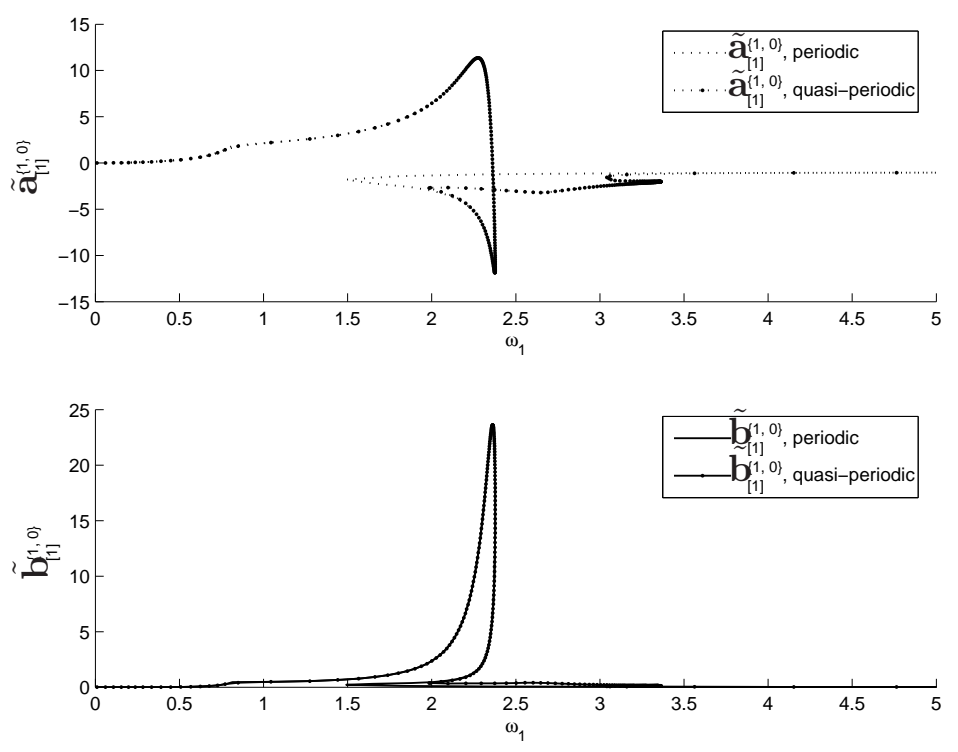

Figure 5: TERM $\{1,0\}$ COEFFICIENTS FOR QUASI-PERIODIC AND PERIODIC EXCITATION

- periodic excitation corresponding to the second term of (35) only.

On Fig. 5, one can remark a discrepancy over the range $\omega_{1} \in[1.5,3.5]$ that highlights the interaction of the principal harmonic components of response: the excitation at $\omega_{2}$ modifies the magnitude of response at $\omega_{1}$. Fig. 6 puts forward analogous dissimilarity due to the influence of the excitation at $\omega_{1}$ on the response at $\omega_{2}$, especially for $\omega_{1} \in[1,2.5]$. As shown on Fig. 7, the composite components are responsible for the secondary resonances at $\omega_{1}=1.3$ and $\omega_{1}=4$ (which correspond to points $\mathrm{A}$ and $\mathrm{E}$ on Fig. 2).

\section{CONCLUSION}

A generalized harmonic balance method using AFT approach and an arc-length continuation is developed. This method is applied to a rotordynamic problem consisted of a modified Jeffcot rotor with a piecewise linear radial stiffness under quasi-periodic excitation by two unbalance-type forces. The response is quasi-periodic and strongly non-linear. It puts forward mutual influence of different components of resonance due to non-linear coupling. Combination resonances are also observed on mixed components of the response. The verification by time marching has shown that with help of continuation procedure, the stable segments of the response curve have been computed as well as the unstable ones. The improvement of the FFT-based AFT procedure has allowed to half the computation time.

\section{References}

[CU81] L.O. Chua and A. Ushida. Algorithms for computing almost periodic stady state response of non-linear systems to multiple input frequencies. IEEE Trans. Circuits and Systems, 28(10):953-971, 1981.

[Ehr98] F.F. Ehrich, editor. Handbook of rotordynamics. Krieger Publishing Company, 1998. 

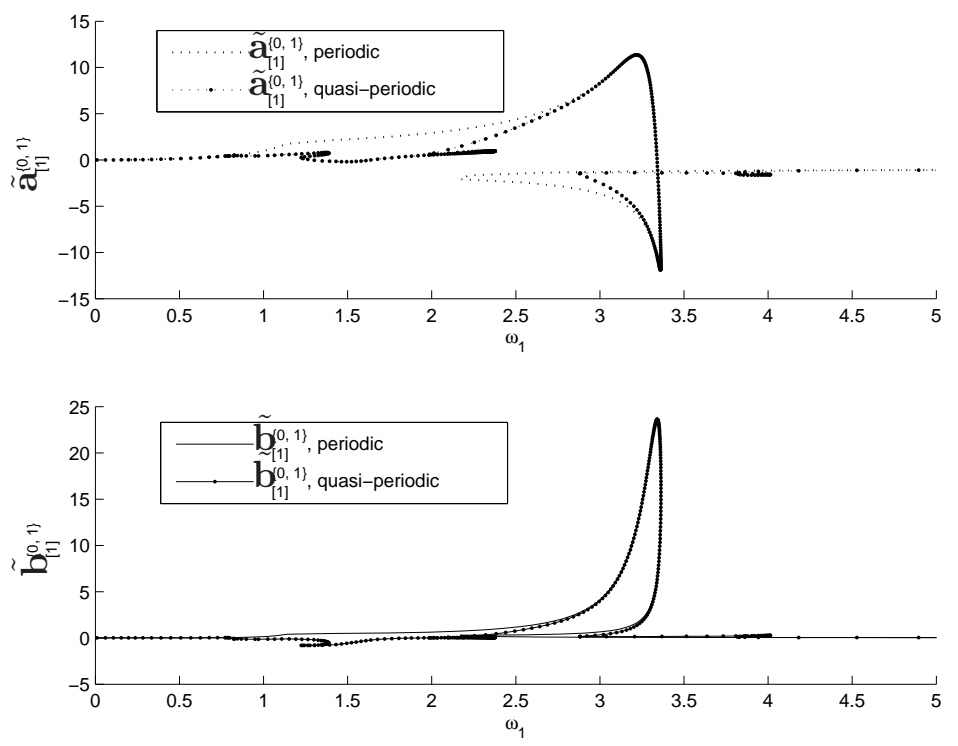

Figure 6: TERM $\{0,1\}$ COEFFICIENTS FOR QUASI-PERIODIC AND PERIODIC EXCITATION
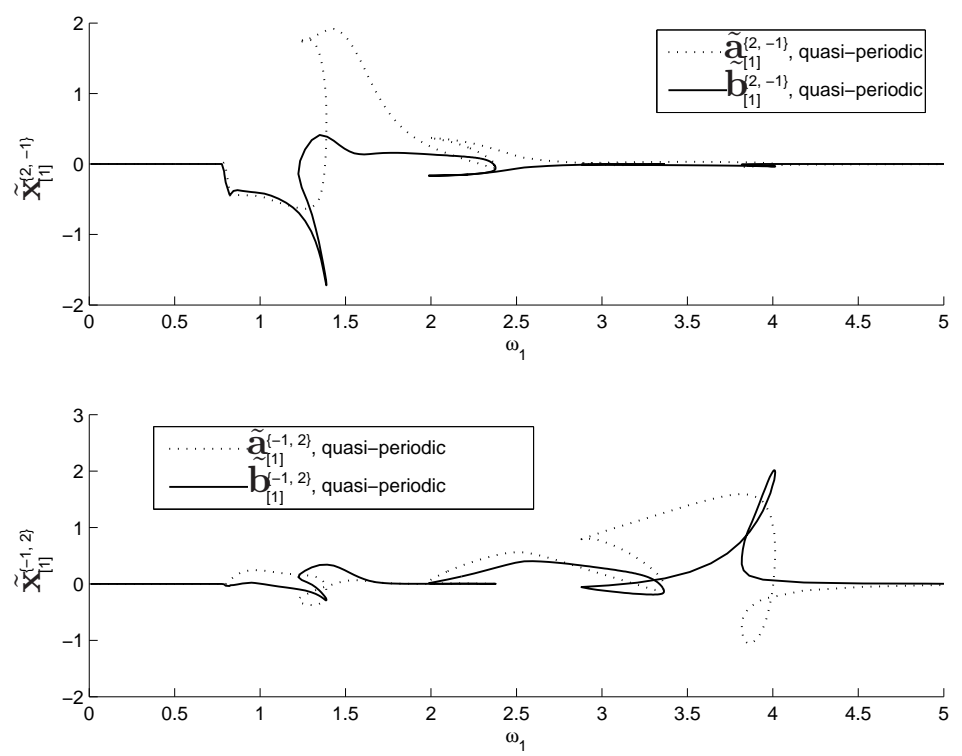

Figure 7: TERMS $\{2,-1\}$ AND $\{-2,1\}$ COEFFICIENTS FOR QUASI-PERIODIC EXCITATION 
[GSTN06] M. Guskov, J.-J. Sinou, F. Thouverez, and O.S. Naraïkin. Numerical and experimental studies of a dynamical response of a dual shaft test rig. Proceedings of IMAC-XXIV Conference on Structural Dynamics, 2006.

[GSTN07] M. Guskov, J.-J. Sinou, F. Thouverez, and O. Naraikin. Experimental and numerical investigations of a dual-shaft test rig with intershaft bearing. International Journal of Rotating Machinery, 2007.

[Har66] T. Harris. Rolling element bearings. John Wiley \& Sons, Inc, 4th edition, 1966.

[Hib75] D.H. Hibner. Dynamic response of viscous-damped multi-shaft jet engines. Journal of Aircraft, 12(4):305-312, 1975.

[KC97] Y.B. Kim and S.-K. CHOI. A multiple harmonic balance method for the internal resonant vibration of a non-linear jeffcot rotor. Journal of sound and vibration, 208(3):745-761, 1997.

[KN96] Y.-B. Kim and S.T. Noah. Quasi-periodic response and stability analysis of a non-linear jeffcot rotor. Journal of sound and vibration, 190(2):239-253, 1996.

[LC83] S.L. Lau and Y.K. Cheung. Incremental harmonic balance method with multiple time scales for aperiodic vibration of nonlinear systems. Journal of applied mechanics, 50:871-876, 1983.

[LF97] M. Lalanne and G. Ferraris. Rotordynamics prediction in engineering. Wiley, 2nd edition, 1997.

[PM97] D.A. Peters and J Morillo. Harmonic balance approach for rotorcraft with both unsteady and unknown rotor speed. Proceedings of the 38th AIAA SDM Conference, Kissimmee, Florida, April 7-10, pages AAIA Paper No. 97-1095, 1997.

[PO04] R.R. Pusenjak and M.M. Oblak. Incremental harmonic balance method with multiple time variables for dynamical systems with cubic non-linearities. International journal for numerical methods in engineering, 59:255-292, 2004.

[SL07] J-J. Sinou and A.W. Lees. A non-linear study of a cracked rotor. European Journal of Mechanics - A/Solids, 26(1):152-170, 2007.

[VST08] C. Villa, J.-J. Sinou, and F. Thouverez. Stability and vibration analysis of a complex flexible rotor system. Communications in nonlinear science and numerical simulation, 13(4):804$821,2008$. 